\title{
Mapeamento das tendências de investigação em Educação a Distância e Elearning, na década 2004-2013: estudo exploratório no contexto português
}

\author{
Mapping of research trends in Distance Education and E-learning, in the decade \\ of 2004-2013: an exploratory study in the Portuguese context \\ Mapeo de las tendencias de investigación en Educación a Distancia y Elearning \\ en la década 2004-2013: un estudio exploratorio en el contexto portugués
}

\section{LINA MORGADO ANGELINA COSTA}

\begin{abstract}
Resumo: $\mathrm{O}$ ensino superior vive complexos momentos de mudança à escala mundial. O desenvolvimento exponencial das tecnologias e, consequentemente, da educação a distância e do elearning, os novos públicos que têm acesso a este nível de ensino, o facto de ser, pela sua natureza, um espaço de inovação na relação e construção do conhecimento, os desenvolvimentos científicos, concretamente na área da educação e das tecnologias, são fatores que têm feito emergir necessidades de análise, de avaliação e de reflexão da produção científica, tornando-as tarefas substanciais da comunidade académica. Com este artigo pretende-se esboçar alguns cenários de futuro na educação a distância e elearning, no ensino superior, olhando para a situação de Portugal no contexto europeu. Foram definidos três momentos de investigação. Num primeiro momento, pretende-se delinear o estado da arte da investigação sobre educação a distância e elearning, no ensino superior, mas ir para além dele, num segundo momento, convocando a discussão e a reflexão dos protagonistas no processo. Num terceiro momento, pretende-se confrontar as perspetivas nacionais com as perspetivas de especialistas europeus neste domínio. Os resultados que agora se apresentam constituem ainda um trabalho em progresso e por isso com resultados preliminares e parciais da primeira fase de investigação.
\end{abstract}

Palavras-chave: educação a distância, elearning, ensino superior, investigação, gestão, inovação

Abstract: Higher education experiments complex moments of change to a
worldwide extent. With the exponential development of technology and,
therefore, the distance education and learning, where a new public has access
to this level of education. Other factors like being, by its nature, a space for
innovation in the relationship and construction of knowledge, scientific
developments, particularly in education and technology, contribute to emerging
needs analysis, evaluation and reflection of the scientific production, making
it the substantial tasks of the academic community. This study aims to outline
scenarios of future in distance education and e-learning, in higher education,
looking at Portugal's position in the European context. It has been defined three
stages of the investigation. Initially, it is intended to outline the state of the
art of research on distance education and e-learning, in higher education, but 
go beyond, in a second moment, summoning discussion and reflection of the protagonists in the process. Thirdly, the prospects of national and European perspectives of specialists in this field will be faced up. Still, in the research process, we present here the global design of the study and preliminary and partial results of phase one of the research.

Keywords: distance education, e-learning, higher education, research projects, management, innovation

Resumen: La enseñanza superior vive complejos momentos de cambio a escala mundial. El desarrollo exponencial de las tecnologías y, por consiguiente, de la educación a distancia y del elearning, los nuevos públicos que tienen acceso a este nivel de enseñanza, el hecho de ser, por su naturaleza, un espacio de innovación en la relación y la construcción del conocimiento, los desarrollos científicos, concretamente en el ámbito de la educación y de las tecnologías, son factores que han hecho emerger necesidades de análisis, de evaluación y de reflexión de la producción científica, haciéndolas tareas sustanciales de la comunidad académica. Con este artículo se pretende esbozar algunos escenarios de futuro en la educación a distancia y e-learning en la educación superior, mirando la situación de Portugal en el contexto europeo. Se definieron tres momentos de investigación. En un primer momento, se pretende delinear el estado del arte de la investigación sobre educación a distancia y elearning, en la enseñanza superior, pero ir más allá de él, en un segundo momento, convocando la discusión y la reflexión de los protagonistas en el proceso. En un tercer momento, se pretende confrontar las perspectivas nacionales con las perspectivas de expertos europeos en este ámbito. Los resultados que ahora se presentan constituyen todavía un trabajo en progreso y por ello con resultados preliminares y parciales de la primera fase de investigación.

Palabras clave: educación a distancia, elearning, enseñanza superior, investigación, gestión, innovación

\section{INTRODUÇÃO}

Mais de uma década após a Declaração de Bolonha na Europa, e das alterações exigidas pelo processo de mudança estrutural e funcional que se lhe seguiu, o ensino superior continua a viver momentos difíceis de mudança no espaço europeu, e concretamente em Portugal.

O desenvolvimento exponencial das tecnologias e, consequentemente, $\mathrm{da}$ educação a distância e do elearning, bem como os novos públicos que têm acesso a este nível de ensino, colocam desafios geradores de inseguranças, de incertezas e de resistências em docentes e estudantes que se cruzam com expetativas, entusiasmo e desejo de inovação.

A confluência das novas necessidades de aprendizagem ao logo da vida com as inovações tecnológicas na comunicação a distância têm pressionado as instituições do ensino superior, obrigando-as a dar resposta a novas necessidades 
e exigências. No entanto, a última década tem sido marcada por factos como uma compreensão teórica insuficientemente alargada e coerente das práticas em educação a distância e elearning, por dificuldade em encontrar sentido no leque de possibilidades que se abrem para tornar a aprendizagem mais eficaz e na confusão concetual resultado da emergência vertiginosa de novas terminologias (virtual, aberta, distribuída, a distância), novas tecnologias e novas audiências.

Os processos de implementação das tecnologias digitais na educação têmse mostrado mais complexos do que era inicialmente esperado e as vantagens da sua utilização não parecem ser claramente evidentes e fundamentadas (ANDERSON, DRON, 2011; GURI-ROSENBLIT, GROS, 2011; GUYER, AYDOGDU, 2016). As expetativas criadas nos anos 90 face à aprendizagem mediada por tecnologias, nomeadamente as que preconizavam a transformação radical dos processos de ensino-aprendizagem e as de que o domínio e controlo deste processo passaria dos docentes para os estudantes, não parecem ter sido completamente concretizadas. Por outro lado, este campo científico denota ainda imaturidade, que se expressa, por exemplo, na confusão terminológica, consequência de poder considerar-se um campo relativamente recente e, portanto, com limites ainda pouco precisos (e.g. SANGRÀ, et al., 2011).

Apesar das tentativas de definir claramente os conceitos em causa (Idem, Ibidem), parece ser impossível, neste momento, encontrar uma definição consensual. Um dos fatores marcantes que contribui para este facto é emergência de novos termos à medida que novas tecnologias e ferramentas se vão desenvolvendo. Outro deles é a distinção difusa dos conceitos de educação a distância e de elearning, utilizados, com alguma frequência, como sinónimos. A confusão terminológica tem impacto nos resultados da investigação e, uma vez que a distância não é um critério para definir elearning, parece importante que, nas situações de investigação, seja claramente identificado o contexto em que ocorre (se em educação a distância se em ensino presencial).

Por outro lado, as questões relacionadas com as gerações de educação a distância têm tido alguma reflexão no contexto das práticas institucionais, tendo-se deslocado o seu foco de uma relação centrada nas tecnologias como foi referido por GOMES (2003) e MORGADO (2004) para uma relação mais focada nas pedagogias (ANDERSON, DRON, 2012).

Também o conceito de educação a distância tem experimentado interpretações diversas e ambíguas. Há mais de uma década que se tornou visível a entrada da educação a distância numa era pós-moderna, caraterizada por grande diversidade de meios, resultado da evolução surpreendente e vertiginosa, num curto espaço de tempo, das tecnologias da comunicação (GARRISON, 2000). Novos conceitos e formas de relação e de construção do conhecimento estão em 
discussão e na ordem do dia como os de «flexibilidade» ou de «abertura» (BATES, 2008). Daqui emergiram novas conceções a que não é estranha uma mudança de paradigma consubstanciada na designação de «educação a distância» em vez de «ensino a distância» (GARRISON, 2003).

Por exemplo, em 2006, a Universidade Aberta, em Portugal, definia o seu modelo pedagógico (PEREIRA, QUINTAS, MORGADO, AMANTE, BIDARRA, 2007), no qual, através de quatro princípios, aprendizagem centrada no estudante, flexibilidade, interação e inclusão digital, se desenha uma «Universidade para o futuro», concebida como «uma instituição permanentemente ligada (...) ao mundo, a todo ele (...) um polo potenciador e dinamizador (...) onde cada um pode encontrar e encontrar-se na construção do conhecimento (...) [que] não se restringe a fornecer informação aos seus estudantes, (...) [mas que se abre] a que estes convoquem a si a construção do saber, partilhando os seus mundos (PEREIRA et al:: 4).

O desenvolvimento tecnológico acima referido permitiu um salto qualitativo no desenvolvimento da educação a distância, nomeadamente nas instituições tradicionais do ensino superior. Com uma procura em crescendo do ensino superior (neste momento refreada pela conjuntura económica à escala global) este novo sistema de gestão das aprendizagens entrou na maior parte destas instituições apesar de configurado em moldes semelhantes aos do ensino presencial. Mas os últimos anos trouxeram contribuições teóricas e reflexivas que exprimem um novo paradigma, recuperando modelos socioconstrutivistas que se concretizam, por exemplo, no quadro conceptual do conectivismo (SIEMENS, 2004; BROUNS et al., 2017) e nas práticas dos Massive Open Online Courses (MOOC).

Relativamente à investigação neste domínio, estudos de meta-análise (e.g. MEANS et al., 2009) apontam debilidades como o tamanho insuficiente das amostras ou o eventual enviesamento dos resultados pela dupla assunção do papel de investigador e de interveniente na situação, sem metodologia apropriada. A estas fragilidades podem acrescentar-se dificuldades em conduzir estudos longitudinais sobre o impacto das tecnologias ou, pelo menos, ter em consideração períodos de tempo mais alargados, ou ainda sair da estrutura convencional de testar o impacto de determinada aplicação/ferramenta.

BATES $(2005 ; 2015)$ afirma que embora a quantidade de investigação neste campo seja considerável, vários estudos apontam para fragilidades na sua qualidade. A circunstância de, maioritariamente, estar baseada num único caso, ser descritiva e qualitativa e não poder contribuir para um quadro teórico sólido, tornam a generalização difícil ou impossível. Mas apesar dessas limitações, começa a emergir um conjunto de resultados que já permitem identificar orientações consistentes para o estabelecimento de práticas de educação a distância e elearning 
bem-sucedidas (BATES, 2017). A questão que aqui merece destaque é que, muitas vezes, os decisores institucionais desconhecem esses dados, o que pode contribuir para explicar o facto de tantas iniciativas neste domínio falharem ou não terem os resultados desejados e/ou esperados.

Analisando a qualidade e coerência dos estudos neste campo, GURIROSENBLI e GROS (2011) apresentam quatro áreas de investigação prioritárias no momento atual: a) administração e gestão, b) infraestruturas tecnológicas, c) pedagogia e d) contexto social. Estas áreas deveriam ser cruzadas com três níveis de análise: micro, meso e macro, defendendo a ideia de que o elearning é um campo científico complexo e multidimensional, e que mudanças numa área ou num nível afetam necessariamente os outros. Daí a necessidade de um modelo concetual que represente esta complexidade e oriente a investigação.

Parece importante então salientar duas ideias-chave: a dificuldade em encontrar uma visão estratégica, ao nível macro (aliás, o nível mais negligenciado de investigação), que oriente as políticas nacionais neste domínio e a dificuldade em ter uma visão clara da relação custos-benefícios de implementação das tecnologias na educação. Neste caso, a implementação das tecnologias deve demonstrar ser um valor acrescentado inequívoco e não apenas por serem «inovadoras» ou por «estarem na moda».

Neste momento, pelo menos duas tendências internacionais da educação a distância e elearning merecem destaque, a saber, a emergência e a consolidação de processos de aprendizagem colaborativa em rede e a disseminação do uso de modelos mistos, b-learning, nas instituições tradicionais de ensino superior (HASAN, LAASER, 2010). Poderão estas tendências ser observadas no ensino superior português? Neste momento, todas as instituições de ensino superior têm algum modelo de ambiente virtual de aprendizagem. No entanto, as estratégias institucionais que fundamentam essas práticas e a sua expansão não parecem claras, nem tão pouco os fatores de sucesso a ter em consideração. A investigação, neste domínio, apresenta-se, normalmente, em duas categorias: estudos de caso e inquéritos por questionário (ACZEL et al., 2006), o que parece insuficiente para essa fundamentação.

O ensino superior é, pela sua natureza, um espaço de inovação na relação com o conhecimento e na sua construção e difusão. O desenvolvimento científico, neste caso no âmbito da educação e das tecnologias, tem feito emergir necessidades de análise, de avaliação e de reflexão da produção científica, tornando-as tarefas importantes da comunidade académica.

Demonstra-se assim, a necessidade de melhor compreender o estado da arte da investigação em educação a distância e elearning, no ensino superior em Portugal, e de prospetivar os seus cenários de futuro. 


\section{ESTUDO EMPÍRICO}

Este artigo apresenta parte dos resultados de um estudo mais vasto que pretende delinear cenários de futuro na educação a distância e elearning, no ensino superior, situando Portugal no contexto europeu. Apresentamos aqui apenas as questões de investigação e os objetivos relacionados com este artigo.

\section{QUESTÕES DE INVESTIGAÇÃO E OBJETIVOS}

A partir da questão de investigação mais geral: Que cenários de futuro se desenham para o ensino superior, no domínio da Educação a Distância e Elearning, em Portugal?, apresentamos neste artigo algumas das subquestões de investigação em análise neste estudo ainda exploratório: 1) Quais são as tendências da investigação em educação a distância e elearning, no ensino superior, em Portugal, na década 2004/2013? e 2) Como é que estas tendências se articulam com as políticas e as práticas no ensino superior, no domínio referido?

Os objetivos de investigação do estudo são os seguintes: a) delinear o estado da arte da investigação sobre educação a distância e elearning no ensino superior, na década 2004-2013 no contexto português; b) identificar perspetivas/ perceções da comunidade académica face à investigação neste domínio e ao seu impacto na inovação e nas políticas de educação a distância e elearning no ensino superior; e c) contribuir para a história da educação a distância e elearning no ensino superior, em Portugal.

\section{METODOLOGIA}

\section{DESIGN DO ESTUDO: FASE DE RECOLHA E ANÁLISE DE $D A D O S$}

A investigação empírica envolve três fases de recolha e de análise de dados mas neste artigo apenas será objeto de análise a primeira fase. Num primeiro momento irá confrontar os dados extraídos sobre o estado da arte da investigação sobre educação a distância e elearning no ensino superior, em Portugal, através de uma metodologia de meta-análise, com a perspetiva da comunidade científica sobre esse domínio, prospetivando cenários de futuro através da metodologia de grupos de discussão. Essas perspetivas serão contrastadas com as perspetivas de especialistas europeus neste domínio, através da metodologia de delphi study.

Assim, na primeira fase do estudo (Fase 1) foi realizada uma extensa e profunda análise documental da produção científica no domínio em causa, 
no período de tempo considerado. Esta fase implicou uma meta-análise não convencional, ou seja, uma «meta-análise qualitativa», de acordo com a perspetiva de CARDOSO $(2007 ; 2008 ; 2012)$ e de CARDOSO, ALARCÃO e CELORICO (2010; 2013). A meta-análise, conjunto de técnicas de análise quantitativa, tem sido utilizada mais frequentemente em domínios como o das ciências da saúde, em revisões sistemáticas da literatura para avaliar conjuntos de estudos independente com caráter quantitativo. Os estudos de revisão sistemática com recurso à metaanálise são frequentemente designados por estudos de meta-análise. Na sequência dos objetivos da investigação, será utilizado um estudo deste tipo, mas com um caráter qualitativo na medida em que se pretende abarcar, inventariar e analisar todos os tipos de estudos produzidos num horizonte temporal definido, dentro de uma temática abrangente, sujeitando os dados a procedimentos de análise de conteúdo e a procedimentos estatísticos descritivos.

Para esta fase, foram identificados os documentos que apresentavam investigação no domínio do nosso estudo. O seu conteúdo foi tratado através de categorias definidas, num processo que os agrupou e reconfigurou. Deste modo, foram realizadas as seguintes ações: a) identificação e recolha dos documentos a analisar; b) análise de conteúdo dos mesmos; c) sistematização da informação em base de dados, com consequente d) tratamento estatístico, que permitiu identificar tendências relativamente a critérios como tema, tipo de investigação e metodologia utilizada, técnicas/ferramentas de recolha dos dados, amostra, técnicas/ferramentas de análise dos dados e conclusões.

O corpus de análise foi constituído por todos os documentos que reportavam investigação desenvolvida em educação a distância e elearning no ensino superior, produzida em Portugal, num período de dez anos, 2004 a 2013, inclusive, sob o formato de dissertações de mestrado e de teses de doutoramento, publicações em periódicos, atas de conferências/seminários, obras completas e capítulos de obras.

\section{PROCEDIMENTO}

A recolha de dados começou pela identificação e registo dos documentos que apresentassem investigação sobre educação e distância e elearning no ensino superior, no período considerado, através de descritores de pesquisa como, por exemplo, «educação a distância», «elearning», «ensino superior» no Repositório Científico de Acesso Aberto de Portugal (RCAAP). Em algum momento, estes descritores foram ampliados, combinados ou substituídos como, por exemplo, «online», «b-learning», «educação a distância», «ensino a distância» ou «aprendizagem a distância», ou «elearning» por «e-learning». 
O registo dos documentos foi realizado em ficha construída para o efeito, e preenchida para cada instituição de ensino superior na qual o documento foi publicado, o que possibilitou uma análise da produção científica por instituição de ensino superior.

Do total de documentos identificados, apresentam-se aqui apenas os dados relativos a uma amostra que compreende as dissertações de mestrado e teses de doutoramento após a defesa da prova. Assim, nesta amostra, foram identificados 97 documentos (cf. Quadro 1).

Posteriormente, foi construída uma base dados com a informação específica referente a cada documento, o que permitiu a extração da informação para posterior confronto e análise. Essa base organiza-se em cinco parâmetros fundamentais: tema, objetivos do estudo, quadros teóricos de referência, metodologias de investigação e contributos.

$\mathrm{Na}$ sequência deste levantamento e registo, e a partir de uma primeira análise que englobou, para cada documento, a) título; b) palavras-chave; c) resumo, foram identificadas sete categorias de codificação (cf. Quadro 2.) relativamente aos temas abordadas nos estudos que constituem o corpus de análise. Esta é, ainda, uma abordagem grosseira que carece de análise mais aprofundada, uma vez que não estão ainda extraídos todos os parâmetros atrás referidos.

\section{RESULTADOS PRELIMINARES}

Os resultados obtidos sobre a análise da produção científica nas instituições de ensino superior relacionadas com a temática em estudo, focada nas dissertações de mestrado e teses de doutoramento sintetiza-se no Quadro 1 que apresenta a distribuição por tipologia e ano de publicação. Verifica-se que são os anos de 2008, 2009 e 2011 e 2012 os anos com maior produção científica dedicada à investigação sobre a temática da educação a distância e elearning no ensino superior.

Numa primeira análise, pode observar-se um aumento progressivo, embora não constante, do número de investigações neste domínio, com um decréscimo substancial no ano de 2010, facto que carece de investigação complementar. No Gráfico 1., que a seguir se apresenta, podemos observar esta evolução em termos gráficos. 


\section{Quadro 1 - Número total de estudos por ano e por tipologia}

\begin{tabular}{|c|c|c|c|}
\hline Anos & $\begin{array}{c}\text { Dissertação } \\
\text { mestrado }\end{array}$ & $\begin{array}{c}\text { Tese } \\
\text { doutoramento }\end{array}$ & Total \\
\hline 2004 & 3 & 1 & 4 \\
\hline 2005 & 3 & 2 & 5 \\
\hline 2006 & 5 & 2 & 7 \\
\hline 2007 & 4 & 4 & 8 \\
\hline 2008 & 8 & 3 & 11 \\
\hline 2009 & 12 & 2 & 14 \\
\hline 2010 & 3 & 3 & 6 \\
\hline 2011 & 10 & 8 & 18 \\
\hline 2012 & 11 & 5 & 16 \\
\hline 2013 & 3 & 5 & 8 \\
\hline Total & 62 & 35 & 97 \\
\hline
\end{tabular}

\section{Gráfico 1 - Representação gráfica do número total de investigações por}

ano e por tipologia

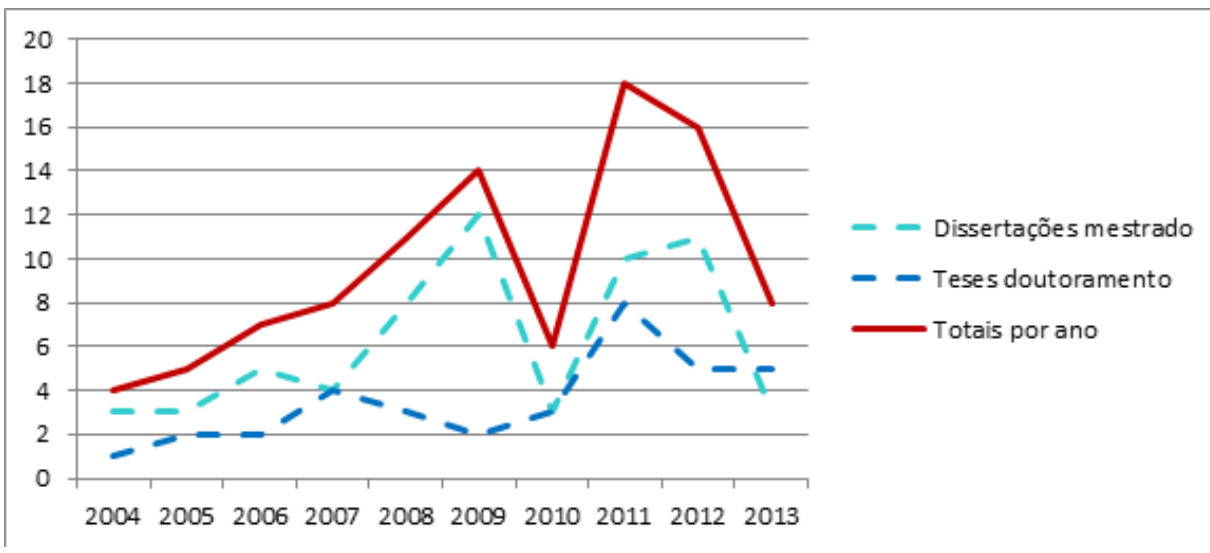

Apresentam-se no Quadro 2, as categorias extraídas a partir dos temas investigados nos documentos analisados que constituíram esta amostra tendo sido cada uma delas previamente definida e descrita para a análise a efetuar.

No que respeita à categoria «Modelos Pedagógicos» incluiu-se, por exemplo, as investigações sobre propostas e modelos de elearning ou de b-learning para determinada instituição de ensino superior. Por sua vez, a categoria «Contextos de Aprendizagem» engloba estudos por exemplo, sobre plataformas de suporte ao processo de ensino-aprendizagem ou, ainda, sobre comunidades virtuais de aprendizagem. 


\section{Quadro 2 - Número total de estudos por tema de investigação e tipologia}

\begin{tabular}{|c|c|c|c|}
\hline Temas & $\begin{array}{c}\text { Dissertações de } \\
\text { mestrado }\end{array}$ & $\begin{array}{c}\text { Teses de } \\
\text { doutoramento }\end{array}$ & Total \\
\hline Modelos pedagógicos & 6 & 5 & 11 \\
\hline $\begin{array}{c}\text { Contextos de } \\
\text { aprendizagem }\end{array}$ & 11 & 11 & 22 \\
\hline $\begin{array}{c}\text { Tecnologias/ } \\
\text { ferramentas/recursos }\end{array}$ & 19 & 11 & 30 \\
\hline $\begin{array}{c}\text { Processos de } \\
\text { comunicação/ } \\
\text { interação }\end{array}$ & 9 & 3 & 12 \\
\hline $\begin{array}{c}\text { Perceções/atitudes/ } \\
\text { expetativas }\end{array}$ & 8 & 1 & 9 \\
\hline Avaliação & 3 & 1 & 4 \\
\hline $\begin{array}{c}\text { Formação } \\
\text { professores/e-tutores }\end{array}$ & 61 & 4 & 9 \\
\hline Total & 56 & 97 \\
\hline
\end{tabular}

No que se refere à categoria «Tecnologias/Ferramentas/Recursos» foram incluídas as investigações que apresentam experiências circunscritas, por exemplo, o uso de videojogos numa unidade curricular específica. Já na categoria «Processos de comunicação/interação» incluíram-se os estudos que analisavam/ avaliavam, por exemplo, estratégias de interação no quadro de um curso ou unidade curricular específicos.

A categoria «Perceções/atitudes/expetativas» abarca as investigações que tiveram por objetivo, por exemplo, analisar graus de satisfação em determinado curso enquanto a categoria «Avaliação» agrega exclusivamente os estudos sobre e-assessment, não estando aqui incluídos estudos sobre avaliação de modelos, contextos ou ferramentas/tecnologias. Finalmente na categoria «Formação de professores/e-tutores» encontram-se os estudos direcionados especificamente para a formação de professores no âmbito da tecnologia ou, por exemplo, o desenvolvimento de competências de e-tutores no quadro do e-learning. O Gráfico 2., que a seguir se apresenta, permite visualizar a distribuição de estudos por tema e por tipologia. 

de investigação e tipologia

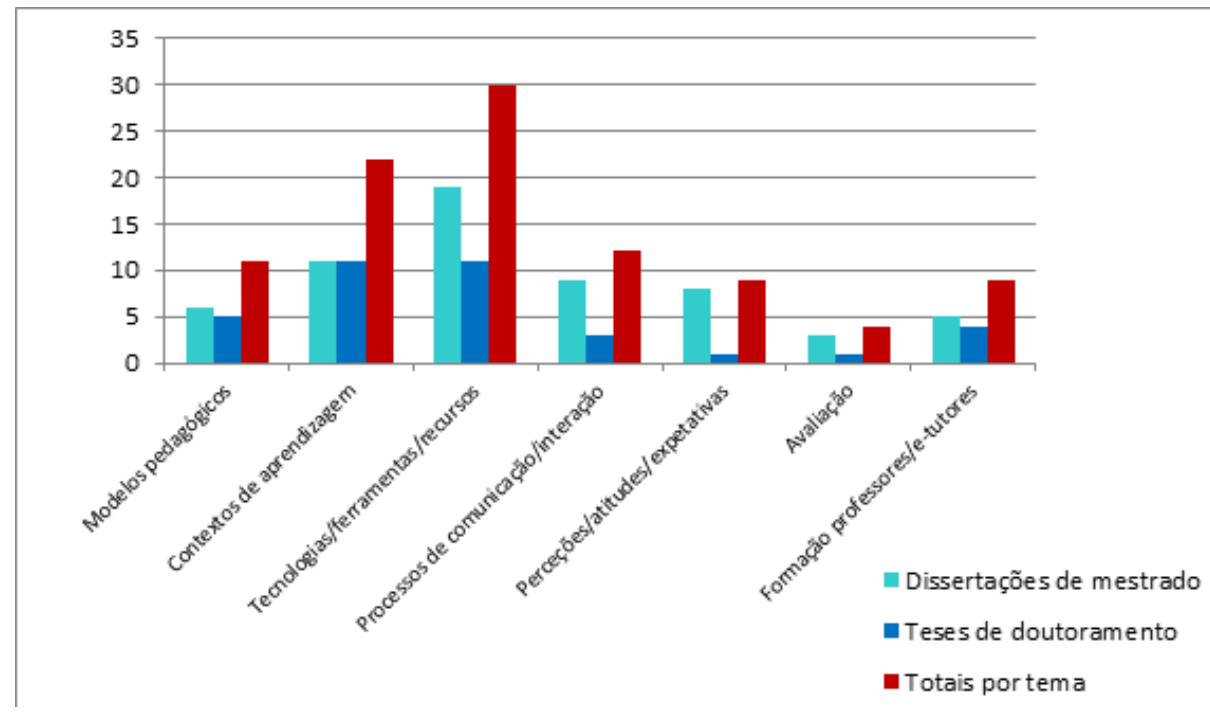

Apesar de ainda constituírem resultados preliminares e numa perspetiva exploratória, sublinha-se que a maior parte dos estudos são descritivos, resultam da observação de situações e são concretizados em estudos de caso de práticas institucionais ou em inquéritos por questionário, indo no sentido daquilo que é apontado pela literatura neste domínio (e.g. ACZEL et al., 2006).

\section{IMPACTO NA GESTÃO DA EDUCAÇÃO A DISTÂNCIA E ELEARNING}

Foram previstas algumas dificuldades no desenvolvimento deste estudo, nomeadamente as relacionadas com a identificação do corpus de análise.

As dificuldades previstas mostraram-se reais, uma vez que os sistemas de bases de dados dos serviços académicos de documentação se mostram incompletos, alguns dos documentos não estão acessíveis e, particularmente, alguns dos mais recentes ainda não deram entrada nos repositórios. Nestas situações, e após a sua identificação, os autores irão ser solicitados, através de correio eletrónico, a facultar uma listagem dos trabalhos desenvolvidos, ou disponibilizar-se-á uma lista organizada, inquirindo-os da sua exaustividade (cf. CARDOSO, 2007).

Os resultados obtidos, ainda que preliminares, apontam para o facto de que a maior parte da investigação desenvolvida nas instituições de ensino superior no quadro de doutoramentos e mestrados, ao longo de um período de uma década 
(2004-2013) sobre a temática da Educação a Distância e eLearning no ensino superior, se situa, maioritariamente, num nível intermédio dos níveis encontrados em outras investigações similares, o Nivel Meso: gestão, organização e tecnologia. Em seguida, a maior frequência registada da investigação realizada situa-se Nivel Micro: ensinar e aprender em educação a distância. Sublinha-se ainda uma tendência de alguns estudos se situarem no Nivel Macro: Sistemas e Teorias de Educação a Distância, mas com menor frequência.

Se tivermos em conta que, por exemplo, entre 2006-2009 se tratou dum período de grande dinâmica de inovação na gestão de educação a distância e, em certa medida disruptivo, com a criação e implementação do um novo modelo pedagógico institucional de Educação a Distância (PEREIRA et al., 2007) configurando novas práticas de gestão da educação a distância pela Universidade Aberta (UAb), pensamos que os resultados obtidos no Nivel Meso podem ser explicados. Esta tendência não é acompanhada nos estudos referidos na literatura nomeadamente os de ZAWACKI-RICHTER (2009) e os de ZAWACKIRICHTER, BÄCKER, VOGT (2009).

Um outro indicador que pode ser interessante cruzar e que explicaria algumas das tendências registadas, seria a matriz dos departamentos responsáveis pelos graus cuja produção científica foi analisada: se departamentos de vocação mais tecnológica, ou de vocação mais pedagógica. As instituições que mais contribuíram para esta análise, foram a Universidade de Aveiro, seguida da Universidade Aberta e Universidade do Minho. Por outro lado, a partir de 2011 tem lugar também uma iniciativa anual, que agrega as instituições de ensino superior e a sua produção ao nível da educação a distância e elearning, o ELIES ${ }^{1}$, traduzindo aqui também o seu impacto no desenvolvimento da investigação.

Relativamente à primeira subquestão de investigação consideramos que foram descritas as principais tendências de investigação dedicadas a educação a distância e Elearning no ensino superior. No que respeita à segunda subquestão é importante dar continuidade às restantes fases do estudo para ser possível cruzar os resultados e poder responder a esta questão.

Este estudo percorre um trajeto conceptual e metodológico original e, portanto, exploratório. A sua concretização poderá concorrer para alargar o espectro da investigação em educação no ensino superior, em Portugal, seguindo as tendências internacionais que solicitam inovação neste domínio. Não se assumindo como um estudo de investigação-ação, nem tendo as suas caraterísticas, pode, no entanto, dar um contributo para a consciencialização da comunidade

1 Encontro de Instituições e Unidades de Elearning do Ensino Superior (ELIES), encontro com periodicidade anual. 
científica sobre as práticas de investigação e sobre o modo como elas são fatores de inovação e de criação de cenários de futuro no domínio da educação a distância e elearning, bem como para os gestores e políticos da área.

\section{REFERÊNCIAS}

ACZEL, J. et al. (2006). Identifying innovation and success factors in higher education elearning strategies. In: Bernath, U. \& Sangrà, A. (Eds). Research on competence development in online distance education and e-learning (141-156). Selected Papers from the 4th EDEN Research Workshop in Castelldefels/Spain Oldenburg: Center for Lifelong Learning, Carl von Ossietzky University.

ALBUQUERQUE, F. (2007). Tecnologias educativas: análise das dissertações de mestrado realizadas em Portugal. Sísifo. Revista de Ciências da Educação, 3, 7-24.

ALMENARA, J. (2008). E-learning: metaanálisis de investigaciones y resultados alcanzados. Ministerio de Educación y Ciencia: Dirección General de Universidades.

ALTBACH, P., REISBERG, L. \& RUMBLEY, L. (2009). Trends in Global Higher Education: Tracking an Academic Revolution. UNESCO 2009 World Conference on Higher Education.

ANDERSON, T., DRON, J. (2012). Três gerações de Pedagogia de Educação a Distância, Revista Científica em Educação a Distância, EAD em Foco, 119-134.

ANDERSON, T. (2005). Distance learning. Social software's killer ap? In Proceedings of the Open \& Distance Learning Association of Australia: Adelaide: ODLAA.

ANDERSON, T. (2006). Higher education evolution: Individual freedom afforded by educational social software. In: M. Beaudoin, Perspetives on higher education in the digital age (77-90). Nova Iorque: Nova Science Publishers.

ANDERSON, T.(Ed.) (2008). Theory and practice of online learning. (2. ${ }^{\mathrm{a}}$ ed.). Edmonton: Athabasca University Press.

ANDERSON, T.\& Kanuka, H. (2003). e-Research: methods, strategies, and issues. Boston: Allyn \& Bacon. 
ANDERSON, T.\& Shattuck, J. (2012). Design-Based Research: A decade of progress in education research? Educational Researcher, 41, 16-25.

BATES, T. (2005). Charting the Evolution of Lifelong Learning and Distance Higher Education: The Role of Research. In C. Macintosh (ed.) Lifelong Learning and Distance in Higher Education (1-18). Paris: UNESCO/Commonwealth of Learning.

BATES; T. (2006). Developing a strategic plan for e-learning in a polytechnic. In M. Bullen \& D. Janes (eds.) Making the Transition to e-Learning: Strategies and Issues. Hershey, PA: Ideas Group.

BATES, T. (2008). Transforming distance education through new technologies. In T. Evans, M. Haughey \& D. Murphy (eds.) The International Handbook of Distance Education (217-235). Bingley: Emerald Press.

BATES, T. (2010). Elearning progress in higher education: The voice of experience. Elearningeuropa, entrevista de Silvia Adriana Tomescu, Librarian, Central University Library Bucharest, Political Science Faculty Library.

BATES, T. (2015). Educar na Era Digital, Versão ABED,

BATES, T. (2017). Q\#2 Educational institutions reorganization in the interconnected world, Entrevista Vídeo, UOC.

BLÄTER, A.; Rapp, J-M.; Solà, C.; Davies, H. \& Teixeira, P. (2013). Portuguese bigher education: a view from the outside. Bruxelas: European University Association.

Brouns, F., Teixeira A., Morgado L., Fano S., Fueyo A., \& Jansen D. (2017). Designing massive open online learning processes: The sMOOC pedagogical framework, Jemni, M., Kinshuk, \& Khribi M. K. (Eds). Open education: From OERs to MOOCs, 315-336, DOI: 10.1007/978-3-662-52925-6_16

CARDOSO, T. (2007). Interaç̧ão verbal em aula de linguas: meta-análise da investigaçãa portuguesa entre 1982 e 2002. (Tese de doutoramento). Universidade de Aveiro, Aveiro, Portugal.

CARDOSO, T.; Alarcão, I. \& Celorico, J. (2010). Revisão da literatura e sistematização do conhecimento. Porto: Porto Editora. 
COLLIS, B. \& Wende, M. (2002). Models of technology and change in higher education. Center for Higher Education and Polices/Faculty of Educational Science and Technology of University of Twente.

CONOLE, G. \& Alevizou, P. (2010). A literature review of the use of Web 2.0 tools in Higher Education. A report commissioned by the Higher Education Academy. The Open University.

CORREIA, L. \& Pinheiro, B. (2012). E-learning: perspetiva histórica de um processo em curso. Historia. Revista da FLUP Porto, IV (2), 195-216.

COUTINHO, C. (2005). ICT in education in Portugal: a review of 15 years of research. International Conference on Education and Information Systems, Orlando, Florida.

COUTINHO, C. (2006a). A investigação em «meios de ensino» entre 1950 e 1980: expectativas e resultados. Revista Portuguesa de Educação, 19 (1), 153-174).

COUTINHO, C. (2006b). Aspectos metodológicos da investigação em tecnologia educativa em Portugal (1985-2000). Braga: Universidade do Minho, CIED, 177-197.

COUTINHO, C., \& Bottentuit Junior, J. (2008). Web 2.0 in portuguese academic community: an exploratory survey. In K. McFerrin; R. Weber; R. Carslen \& A. Willis (eds.) Proceedings of the 19th International Conference of the Society for Information Technology \& Teacher Education, SITE 2008, Las Vegas, 1992-1999.

COUTINHO, C., \& Gomes, (2006). Critical review of research in educational technology in Portugal (2000-2005). World Conference on Educational Multimedia, Hypermedia \& Telecommunications, ED-MEDIA 2006, Orlando, Florida.

FANDIÑO, L. (2008). Las redes de investigación virtuales: propuesta de fomento y desarrollo de la cultura investigativa en las instituciones de educación superior. Revista de Universidady Sociedad del Conocimiento, 4 (2), 1-11.

GARRISON, R.; Anderson, T. \& Archer, W. (2010). The first decade of the community of inquiry framework: A retrospective. The Internet and Higher Education 13, (1-2), 5-9. 
GARRISON, D. \& Anderson, T. (2003). E-learning in the 21st century. Londres: Routledge.

GOMES, M.; Coutinho, C.; Guimarães, F.; Casa-Nova, M. \& Caires, S. (2011). Distance learning and e-learning in Portugal: a study of the perceptions, concepts and teaching practices in the Institute of Education: University of Minho. Proceedings of EDULEARN11 Conference, Barcelona, 2618-2623.

GOMES, M. J. (2003). Gerações de Inovação Tecnológica no Ensino a Distância, Revista Portuguesa de Educação 16, 1: 137 - 156

GURI-ROSENBILT, S. \& Gros, B. (2011). E-learning: confusing terminology, research aps and inherent challenges. Journal of Distance Education, 25 (1).

GUYER, Tolga; AYDOGDU, Şeyhmus A Classification Model and an Open e-Learning System Based on Intuitionistic Fuzzy Sets for Instructional Design Concepts, Journal of Educational Technology Systems, Vol 45, 1, pp. 137 - 160

HASAN, A.; Bielschowsky, C.; Laaser, W.; Mason, R. \& Sangrá, A. (2009). Reforming distance learning higher education in Portugal. Report prepared for the ministry of science, technology and higher education, Portugal.

HASAN, A. \& Laaser, W. (2010). Higher education distance learning in Portugal. State of the art and current policy issues. European Journal of Open, Distance and E-Learning 1, 1-10.

HSU, C.-C. \& Sandford, B. (2007). The delphi Technique: making sense of consensus. Practical Assessment Research \& Evaluation, 12 (10).

JOHNSON, L.; Adams Becker, S.; Cummins, M.; Estrada, V.; Freeman, A. \& Ludgate, H. (2013). NMC Horizon Report: 2013 Higher Education Edition. Austin, Texas: The New Media Consortium.

JORGE, I. (2010). Comunicação mediada por computadores em ambientes de aprendizagem formal: tendências da investigação: de Henri, 1991 a Fini \& Molino, Maio de 2010. In F. Costa, G. Miranda, J. Matos, I. Chagas \& E. Cruz (Eds). Actas do I Encontro Internacional TIC e Educaşão: TICeduca 2010. Lisboa: Instituto de Educação da Universidade de Lisboa. 
LEE, Y., Driscoll, M. P., \& Nelson, D. W. (2004). The past, present, and future of research in distance education: Results of a content analysis. American Journal of Distance Education, 18(4), 225-241.

MCGREAL, R.; Kinuthia, W. \& Marshall, S. (Eds.) (2013). Open educational resources: innovation, research and practice. Vancouver: Commonwealth of Learning and Athabasca University.

MEANS, B.; Toyama, Y.; Murphy, R.; Bakia, M. \& Jones, K. (2010). Evaluation of evidence-based practices in online learning: a meta-analysis and review of online studies. Washington: U.S. Department of Education, Office of Planning, Evaluation, and Policy Development.

MEEK, V.; Teichler, U. \& Kearney, M-L. (Eds.). (2009). Higher education, research and innovation: changing dynamics. Report on the UNESCO Forum on higher education, research and knowledge 2001-2009. Kassel: International Centre for Higher Education Research Kassel.

MORGADO, L. (2004). Ensino Online: Contextos e Interações, Tese de Doutoramento em Ciências da Educação, Lisboa: Universidade Aberta.

OKOLI, C. \& Pawlowski, S. (2004). The delphi method as a research tool: an example, design considerations and applications. Information \& Management, 42, 15-29.

PÉREZ-SÁNCHEZ, R. \& Víquez-Calderón, D. (2009). Los grupos de discusión como metodología adecuada para estudiar las cogniciones sociales. Actualidades en Psicología, 23-24: 87-101.

PEREIRA, A., QUINTAS, A., MORGADO, L., AMANTE, L. e BIDARRA, J. (2007). Modelo Pedagógico Virtual da Universidade Aberta, uma universidade para o futuro. Lisboa: Universidade Aberta.

PHIPPS, R. \& Merisotis, J. (1999). What's the difference? A review of a contemporary research of the effectiveness of distance learning in highereducation. The Institute for Higher Education Policy, Washington, DC. Retirado de: 
SANGRÀ, A. (2003). La educación a distancia como factor clave de innovación en los modelos pedagógicos. Discursos. Perspectivas em Educação. Universidade Aberta, N. ${ }^{0} 1,15-22$.

SANGRÀ \& Duart, J. (2000). Formación universitaria por medio de la web: un modelo integrador para el aprendizaje superior. In: J. Duart \& A. Sangrà (eds), Aprender en la virtualidad. Barcelona: Gedisa.

SANGRÀ \& Sanmamed, M. (Coords.) (2004). La transformación de las universidades a través de las TIC: discursos y prácticas. Barcelona: UOC.

SANGRÀ; Vlachopoulos, D., Cabrera, N., Bravo, S. (2011). Hacia una definición inclusiva del e-learning. Barcelona: eLearn Center.

SANGRÀ; Vlachopoulos, D. \& Cabrera, N. (2012). Building an inclusive definition of e-learning: an approach to the conceptual framework. IRRODL, 13 (2), 145-159.

SANTOS, C. (2008). O grupo de discussão e os estudos sociológicos em contexto escolar. VI Congresso Português de Sociologia. Mundos sociais: saberes e práticas. Lisboa: Faculdade de Ciências Sociais e Humanas da Universidade de Lisboa.

TRINDADE, A. (2005). The transformation of higher education: convergence of distance and presence learning paradigms. Educação a distância: percursos. Lisboa: Universidade Aberta, 303-321.

ZAWACKI-RICHTER, O. (2009). Research Areas in Distance Education: A Delphi Study. International Review of Research in Open and Distance Learning, 10 (3).

ZAWACKI-RICHTER, O; Bäcker, E. \& Vogt, S. (2009). Review of Distance Education Research (2000 to 2008): Analysis of Research Areas, Methods, and Authorship Patterns. International Review of Research in Open and Distance Learning, 10 (6), 21-50.

LINA MORGADO é doutorada em Educação (Educação Online) é professora no Departamento de Educação e Ensino a Distância da Universidade Aberta de Portugal onde é docente e coordenadora do Mestrado em Pedagogia do eLearning. É investigadora no Laboratório de Educação a Distância e eLearning onde desenvolve investigação na área dos modelos e práticas de Educação a Distância e eLearning na sociedade em rede.E-mail: lina.Morgado@uab.pt 
ANGELINA COSTA é psicóloga e docente de Psicologia. É doutoranda na especialidade de Educação a Distância e eLearning na Universidade Aberta de Portugal. É investigadora do Laboratório de Educação a Distância e eLearning onde desenvolve investigação sobre cenários de futuro, no campo da Educação a Distância e eLearning. E-mail: angelina.costaa@gmail.com

Recebido em março de 2018 Aprovado em abril de 2018 